\title{
Nano-indentation and avalanches in compressed porous $\mathrm{SiO}_{2}$
}

Cite as: Appl. Phys. Lett. 115, 071902 (2019); https://doi.org/10.1063/1.5117499

Submitted: 01 July 2019 . Accepted: 17 July 2019. Published Online: 13 August 2019

Tobias Beirau, and Ekhard K. H. Salje

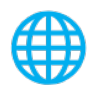

\section{ARTICLES YOU MAY BE INTERESTED IN}

Electrically tuneable exciton energy exchange between spatially separated 2-dimensional semiconductors in a microcavity

Applied Physics Letters 115, 071103 (2019); https://doi.org/10.1063/1.5110492

Remarkable p-type activation of heavily doped diamond accomplished by boron ion implantation at room temperature and subsequent annealing at relatively low temperatures of 1150 and $1300^{\circ} \mathrm{C}$

Applied Physics Letters 115, 072103 (2019); https://doi.org/10.1063/1.5111882

Electrically pumped semiconductor laser with low spatial coherence and directional emission

Applied Physics Letters 115, 071101 (2019); https://doi.org/10.1063/1.5109234
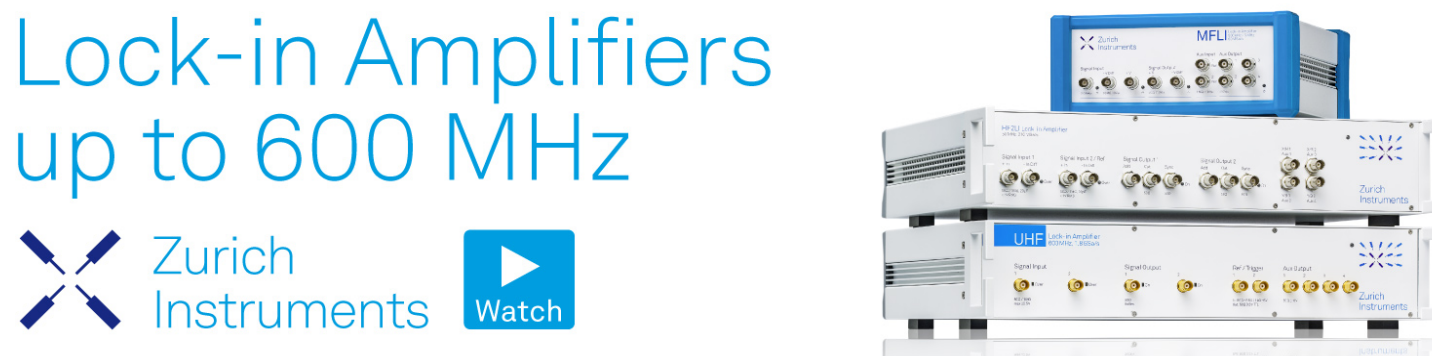


\title{
Nano-indentation and avalanches in compressed porous $\mathrm{SiO}_{2}$
}

\author{
Cite as: Appl. Phys. Lett. 115, 071902 (2019); doi: 10.1063/1.5117499 \\ Submitted: 1 July 2019 . Accepted: 17 July 2019 . \\ Published Online: 13 August 2019

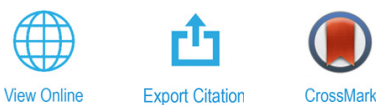

Tobias Beirau ${ }^{1, a)}$ and Ekhard K. H. Salje ${ }^{2, a)}$

\begin{abstract}
AFFILIATIONS
${ }^{1}$ Institute of Geosciences and Geography, Mineralogy/Geochemistry, Martin Luther University Halle-Wittenberg, O6120 Halle, Germany

${ }^{2}$ Department of Earth Sciences, University of Cambridge, Cambridge CB2 3EQ, United Kingdom
\end{abstract}

a)Electronic addresses: tobias.beirau@geo.uni-halle.de and es10002@cam.ac.uk

\begin{abstract}
The "nano-indentation continuous stiffness measurement technique" has been employed to analyze the failure dynamics of mesoporous $\mathrm{SiO}_{2}$ based material (Vycor). The depth dependence of the indentation hardness $(H)$, elastic modulus $(E)$, and elastoplastic parameter $\left(S^{2} / P\right)$ shows crackling noise, which has been analyzed to monitor the jerky strain release. The noise is power law distributed with exponents near $\sim 1.5$ over several decades, confirming avalanche criticality. This value is in good agreement with literature results obtained by other techniques and with earthquake statistics.
\end{abstract}

Published under license by AIP Publishing. https://doi.org/10.1063/1.5117499

The breakdown and collapse behavior of porous structures due to external pressure and deformation is of great importance. Among others, porous materials have been the object of various studies: e.g., for nuclear materials ${ }^{1}$ and $\mathrm{CO}_{2}$ capture and storage, ${ }^{2}$ as models for studying the effects and the mechanisms prior and during earthquakes, ${ }^{3}$ and the collapse of ultralight materials ${ }^{4}$ and artificial and natural formations (e.g., mines, buildings, and bones) $)^{5-8}$ A general better understanding of the occurring phenomena helps to increase the precision of forecasting collapse events. During compression (well before the final breakdown) of porous materials, sudden changes of the internal strain field have been found, seen as discontinuities of the stress displacements. They do not occur continuously, but as coupled events in avalanches leading to crackling noise. Under slow loading, many systems generate crackles, ${ }^{9}$ e.g., our earth during earthquakes, ${ }^{3,10}$ a sheet of paper while crumpling, ${ }^{11}$ ferroic materials under electric and magnetic fields as a result of domain wall movements, ${ }^{12-15}$ metals and alloys during martensitic phase transitions ${ }^{16,17}$ and plastic deformation, ${ }^{4,18}$ and even steel cantilevers as parts of ultrasensitive gravitational wave detectors. ${ }^{19}$ An effective way to detect such abrupt strain field variations, the "jerks," is to measure the related acoustic emission (AE) events. ${ }^{8,10}$ The underlying physical process is the formation of avalanches, seen as sequences of jerks. ${ }^{20}$

Nano-indentation has been successfully used as a complementary method to investigate avalanches in metallic glasses and metals. ${ }^{18,21-23}$ In this study, we extend this approach. We employ the so-called "continuous stiffness measurement (CSM) technique"24-26 to identify avalanches in porous materials during indentation. CSM has been used, e.g., by Greer and $\mathrm{Nix}^{27}$ to investigate size effects in gold. In this technique, a small dynamic oscillation is imposed on the applied force or displacement, and the amplitude and phase of the corresponding signal are measured with a lock-in amplifier. This dynamic measurement differs greatly from the static indentation method in that all parameters are determined as dynamical response functions at a frequency of $100 \mathrm{~Hz}$.

The indentation hardness $(H)$, elastic modulus $(E)$, and elastoplastic parameter $\left(S^{2} / P\right)$ are functions of the indentation depth and monitor the collapse behavior ( jerks) of the mesoporous synthetic silica $\left(\mathrm{SiO}_{2}\right)$ glass "Vycor" (Corning, Inc., New York) during penetration. The maximum likelihood (ML) method ${ }^{28-31}$ was used to characterize the jerk spectra superposed to $H, E$, and $S^{2} / P$.

The sample was a polished plane-parallel plate of Vycor. This material is a nongranular structure composed of a continuous glass skeleton with a porosity $(\Phi)$ of 0.4 and an average pore diameter of $7.5 \mathrm{~nm}^{32}$ The mechanical properties were measured with a Nanomechanics iNano nanoindenter, equipped with a diamond Berkovich indenter tip that operates in a continuous stiffness mode (CSM). Using CSM, the sample stiffness $(S)$ is measured continuously during loading of the indenter. ${ }^{24-26}$ Fused silica was the reference material for calibration. The instrument software calculated the hardness $(H)$ and the elastic modulus $(E)$ during the loading process according to Oliver and $\mathrm{Pharr}^{24}$ with 


$$
H=\frac{P}{A},
$$

where $P$ is the imposed load and $A$ the projected contact area between the indenter and the sample determined from the shape of the indenter and using the Oliver and Pharr method. ${ }^{25,33}$ The measured contact stiffness $(S)$ is related to the elastic properties of both the sample and indenter and the contact area through ${ }^{34}$

$$
S=\beta \frac{2}{\sqrt{\pi}} E_{r} \sqrt{A}
$$

where $\beta$ is a constant depending on the indenter geometry (for Berkovich geometry $\beta=1.05)^{25}$ and $E_{r}$ is the reduced modulus given by

$$
\frac{1}{E_{r}}=\frac{\left(1-\nu_{i}^{2}\right)}{E_{i}}+\frac{\left(1-\nu^{2}\right)}{E},
$$

where $E_{i}$ and $\nu_{i}$ are the elastic modulus and Poisson's ratio of the indenter, respectively (for the diamond indenter, $E_{i}=1141 \mathrm{GPa}$ and $\left.\nu_{i}=0.07\right)$. $E$ and $\nu$ are the elastic properties of the sample. $E_{r}$ considers that elastic displacements take place in the sample and in the indenter. ${ }^{25}$ A Poisson's ratio of 0.188 and a target indentation strain rate of $0.01 \mathrm{~s}^{-1}$ have been used. Values of several indents were analyzed over an indentation depth of 200-1400 nm. Additionally, the material's resistance to plastic penetration $\left(P / S^{2}\right)^{33,35}$ has been used. It is based on the directly measured parameters, load $(P)$ and contact stiffness $(S)$, and is therefore independent of the contact area $(A)$ of the indenter. $P / S^{2}$ is directly proportional to $H$ and $1 / E_{r}^{2}$ expressed by ${ }^{24,25,35}$

$$
\frac{P}{S^{2}}=\frac{1}{\beta^{2}} \frac{\pi}{4} \frac{H}{E_{\mathrm{r}}^{2}} .
$$

$P / S^{2}$ is known to give a good approximation for $H / E_{r}^{2}$ for materials with a significantly lower modulus than diamond, ${ }^{33}$ which is the case for Vycor. In this study, we use the inverse parameter $S^{2} / P$, the elastoplastic factor.

The maximum likelihood estimation (MLE) method ${ }^{28}$ has been used to analyze the jerk spectra ${ }^{8,29-31}$ by

$$
\varepsilon\left(x_{\min }\right)=1+n\left[\sum_{i=1}^{n} \ln \frac{x_{i}}{x_{\min }}\right]^{-1},
$$

with $x_{i}, i=1, \ldots, n$, being the observed values of $x$ such that $x_{i} \geq x_{\min }$, while $\varepsilon$ (defined as a positive quantity) is the exponential parameter indicated by a plateau in the results of the analysis. The standard error $^{28}$ is expressed as

$$
\sigma=\frac{\varepsilon\left(\mathrm{x}_{\min }\right)-1}{\sqrt{\mathrm{n}}}+\mathrm{O}\left(\frac{1}{\mathrm{n}}\right) .
$$

From nano-indentation measurements, we obtained an average elastic modulus for Vycor of $\sim 17.2 \pm 0.4 \mathrm{GPa}$ in excellent agreement with manufacturers specifications $\left(2.5 \times 10^{6} \mathrm{psi}\right)$ and with literature data. $^{36}$ Our value is also close to that calculated from Resonant Ultrasound spectroscopy $(15.8 \mathrm{GPa})$ by Koppensteiner et al. ${ }^{32} \mathrm{We}$ measured an average indentation hardness of $\sim 1.57 \pm 0.04 \mathrm{GPa}$ and an elastoplastic parameter of $\sim 249.9 \pm 9 \mathrm{GPa}$. $E$ is directly related to interatomic bonding and $H$ denotes the resistance to plastic deformation, while the directly measurable $S^{2} / P$ is connected to both via Eq. (4). These mechanical parameters depending on the internal structure have been used to monitor the ongoing volume collapse of Vycor caused by the penetration of the indenter tip.

The depth evolution of $H, E$, and $S^{2} / P$ is shown in Fig. 1 . The jerk spectra were constructed as the square of the first derivative of the primary depth dependences. No smoothing of the original data was used. The jerks are hence a measure for the energy change $\sim(\delta H)^{2},(\delta E)^{2}$,
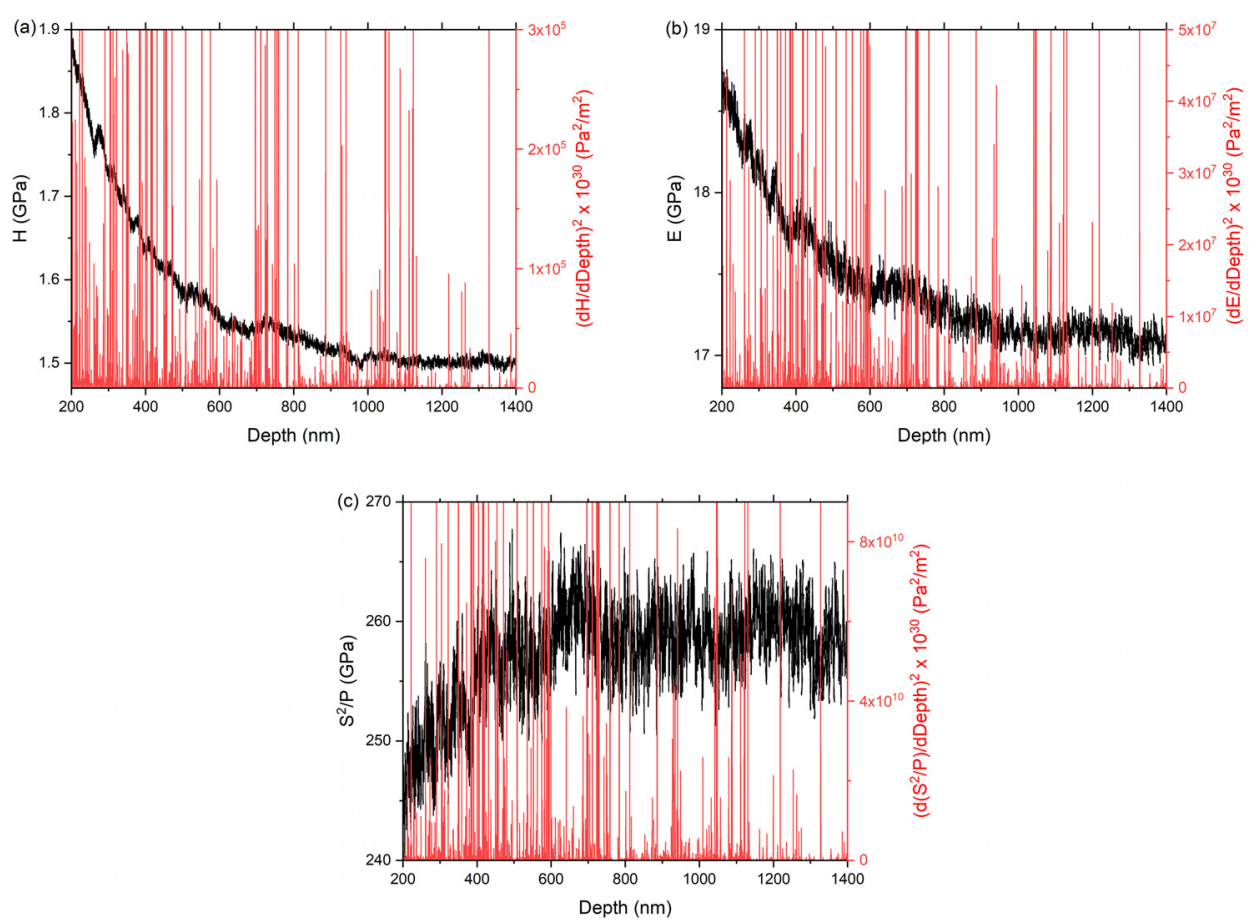

FIG. 1. Depth evolution of (a) $H$, (b) $E$, and (c) $S^{2} / P$ with the related squares of their derivatives (jerk-spectra). The total number of recorded signals is $\mathrm{N}=22552$. 

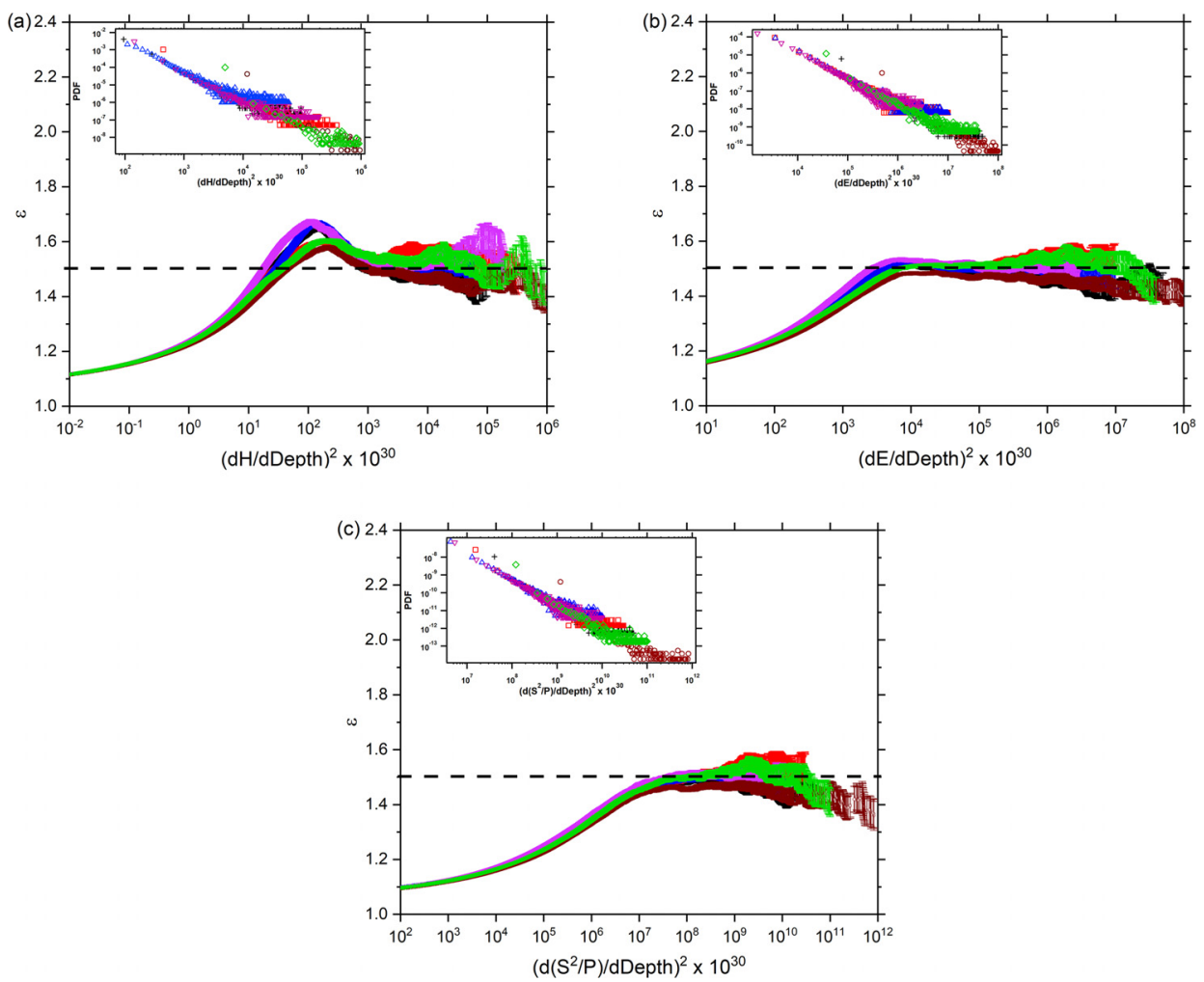

FIG. 2. ML analysis of the squares of the derivate of (a) $H$, (b) $E$, and (c) $S^{2} I P$ after depth for six exemplary indents (each symbol/curve relates to an independent experiment), defining an exponent around 1.5 (dashed line). Insets: probability distribution functions of avalanche energies showing good power law dependences.

and $\left(\delta S^{2} / P\right)^{2}$, per unit depth change. ${ }^{37}$ Deng et al. ${ }^{38}$ have already simulated such behavior where sudden drops in hardness are indeed not smooth curves but contain jerks and subjerks during the drop. The method of identifying jerks as the squares of the slopes includes these effects and is hence more reliable than the registration of simple drop spectra. The review of Hardiman et al. ${ }^{39}$ provides further details. Yield points were observed in $\mathrm{GaAs}$ and $\mathrm{Fe}-3$ wt. $\% \mathrm{Si}$ single crystals when surfaces were indented. ${ }^{40}$ The closeness of our results to the energy exponents in mean field theory (1.33-1.6) is relevant to identify the physical origin of the jerk spectra. Similarly, Budrikis et al. ${ }^{41}$ have explored indentation in a theoretical model and concluded that the size distribution of avalanches follows a much smaller exponent of 1.29 which can be estimated to relate to an energy exponent well below the mean field limit. A full analysis of the atomic mechanisms and thereby the exact identification of the power law exponents, namely, "energy" or "size," is intended by future molecular dynamics simulation. The effect of the squaring of the slopes has already been simulated. ${ }^{20}$ It was shown that the statistical analysis is not significantly influenced, while the statistics of the dataset is much improved for the squared datasets (see the supplementary material).

Figure 2 depicts the ML analysis of six indents. The depth evolution of $(d H / d D e p t h)^{2},(d E / d D e p t h)^{2}$, and $\left[d\left(S^{2} / P\right) / d D e p t h\right]^{2}$ indicates flat plateaus over around 3 decades that define an exponent around 1.5. This exponent is in good agreement with previous measurements using a Dynamical Mechanical Analyzer (DMA), ${ }^{30,31}$ which provide a similar energy exponent from fitting of the power law distributions and a slightly higher value of $\varepsilon \sim 1.7$ from MLE. Our value is close to that from AE spectroscopy (1.39). ${ }^{5,42}$ In comparison with mean field theory, our experimental exponent lies between the limits 1.33 and 1.667 for fast and force integrated jerks, respectively. ${ }^{10,43}$ From the view of earthquake statistics, ${ }^{3}$ our exponent is in excellent agreement with that suggested by Kagan. ${ }^{44}$ The histograms of the probability distribution function (PDF) of the binned jerks are shown as log-log plots (see insets in Fig. 2).

In summary, we showed that the nano-indentation in Vycor using continuous stiffness measurements is suitable to detect avalanches in porous materials. The mechanical properties (i.e., indentation hardness, elastic modulus, and elastoplastic parameter) as a function of depth are sensitive parameters to monitor the chain reactions of collapsing pores during indentation. $E$ directly mirrors the breaking of bonds and provides the best defined plateau. Using the maximum likelihood method, we obtain an exponent $\sim 1.5$ that is in good agreement with literature data for energy exponents in Vycor. We found mean-field behavior in good approximation. In addition, our measurements provide further evidence for the strong similarity between the failure of porous Vycor and earthquake statistics. If it criticality represents the "interface" between ordered and disordered states, ${ }^{45}$ nano-indentation CSM is an excellent technique (related to its small scale) to drive a system to several critical points without provoking a complete breakdown of the material.

See the supplementary material for the exemplary effect of the squaring of the slopes.

Financial support from the Deutsche Forschungsgemeinschaft DFG (No. BE 5456/2-1) is gratefully acknowledged (T.B.). E.K.H.S. is grateful to EPSRC for support (No. EP/P024904/1). 


\section{REFERENCES}

${ }^{1}$ P. Makowski, X. Deschanels, A. Grandjean, D. Meyer, G. Toquer, and F. Goettmann, "Mesoporous materials in the field of nuclear industry: Applications and perspectives," New J. Chem. 36, 531 (2012).

${ }^{2}$ A.-H. Lu and S. Dai, Porous Materials for Carbon Dioxide Capture (Springer, Berlin/Heidelberg, 2014).

3J. Baró, Á. Corral, X. Illa, A. Planes, E. K. H. Salje, W. Schranz, D. E. SotoParra, and E. Vives, "Statistical similarity between the compression of a porous material and earthquakes," Phys. Rev. Lett. 110, 088702 (2013).

${ }^{4}$ Y. Chen, X. Ding, D. Fang, J. Sun, and E. K. H. Salje, “Acoustic emission from porous collapse and moving dislocations in granular Mg-Ho alloys under compression and tension," Sci. Rep. 9, 1330 (2019).

${ }^{5}$ E. K. H. Salje, D. Soto-Parra, A. Planes, E. Vives, M. Reinecker, and W. Schranz, "Failure mechanism in porous materials under compression: Crackling noise in mesoporous $\mathrm{SiO}_{2}$," Philos. Mag. Lett. 91, 554 (2011).

${ }^{6}$ F. Kun, I. Varga, S. Lennartz-Sassinek, and I. G. Main, "Rupture cascades in a discrete element model of a porous sedimentary rock," Phys. Rev. Lett. 112, 065501 (2014).

7J. Baró, P. Shyu, S. Pang, I. M. Jasiuk, E. Vives, E. K. H. Salje, and A. Planes, "Avalanche criticality during compression of porcine cortical bone of different ages,” Phys. Rev. E 93, 053001 (2016).

${ }^{8}$ X. Jiang, D. Jiang, J. Chen, and E. K. H. Salje, "Collapsing minerals: Crackling noise of sandstone and coal, and the predictability of mining accidents," Am. Mineral. 101, 2751 (2016).

${ }^{9}$ J. P. Sethna, K. A. Dahmen, and C. R. Myers, "Crackling noise," Nature 410, 242 (2001).

${ }^{10}$ E. K. H. Salje and K. A. Dahmen, "Crackling noise in dis-ordered materials," Annu. Rev. Condens. Matter Phys. 5, 233 (2014).

${ }^{11} \mathrm{P}$. A. Houle and J. P. Sethna, "Acoustic emission from crumpling paper," Phys. Rev. E 54, 278 (1996).

${ }^{12}$ S. Zapperi, P. Cizeau, G. Durin, and H. E. Stanley, "Dynamics of a ferromagnetic domain wall: Avalanches, depinning transition, and the Barkhausen effect," Phys. Rev. B 58, 6353 (1998).

${ }^{13} \mathrm{~S}$. Zapperi, C. Castellano, F. Colaiori, and G. Durin, "Signature of effective mass in crackling-noise asymmetry,” Nat. Phys. 1, 46 (2005).

${ }^{14} \mathrm{~F}$. Colaiori, "Exactly solvable model of avalanches dynamics for Barkhausen crackling noise," Adv. Phys. 57, 287 (2008).

${ }^{15}$ E. K. H. Salje, D. Xue, X. Ding, K. A. Dahmen, and J. F. Scott, "Ferroelectric switching and scale invariant avalanches in $\mathrm{BaTiO}_{3}$," Phys. Rev. Mater. 3, 014415 (2019).

${ }^{16}$ E. K. H. Salje, J. Koppensteiner, M. Reinecker, W. Schranz, and A. Planes, "Jerky elasticity: Avalanches and the martensitic transition in $\mathrm{Cu}_{74.08} \mathrm{Al}_{23.13} \mathrm{Be}_{2.79}$ shape-memory alloy,” Appl. Phys. Lett. 95, 231908 (2009).

${ }^{17}$ E. Vives, J. Baró, M. C. Gallardo, J. M. Martín-Olalla, F. J. Romero, S. L. Driver, M. A. Carpenter, E. K. H. Salje, M. Stipcich, R. Romero, and A. Planes, "Avalanche criticalities and elastic and calorimetric anomalies of the transition from cubic Cu-Al-Ni to a mixture of $18 \mathrm{R}$ and $2 \mathrm{H}$ structures," Phys. Rev. B $\mathbf{9 4}$, 024102 (2016).

${ }^{18}$ D. M. Dimiduk, C. Woodward, R. LeSar, and M. D. Uchic, "Scale-free intermittent flow in crystal plasticity," Science 312, 1188 (2006).

${ }^{19} \mathrm{G}$. Vajente, "Crackling noise in advanced gravitational wave detectors: A model of the steel cantilevers used in the test mass suspensions," Phys. Rev. D 96, 022003 (2017).

${ }^{20}$ X. He, X. Ding, J. Sun, and E. K. H. Salje, "Parabolic temporal profiles of nonspanning avalanches and their importance for ferroic switching," Appl. Phys. Lett. 108, 072904 (2016)

${ }^{21}$ X. L. Bian, G. Wang, K. C. Chan, J. L. Ren, Y. L. Gao, and Q. J. Zhai, "Shear avalanches in metallic glasses under nanoindentation: Deformation units and rate dependent strain burst cut-off," Appl. Phys. Lett. 103, 101907 (2013).

${ }^{22}$ S. H. Chen, K. C. Chan, G. Wang, F. F. Wu, L. Xia, J. L. Ren, J. Li, K. A. Dahmen, and P. K. Liaw, "Loading-rate-independent delay of catastrophic avalanches in a bulk metallic glass," Sci. Rep. 6, 21967 (2016).
${ }^{23}$ J. P. Coleman, F. Meng, K. Tsuchiya, J. Beadsworth, M. LeBlanc, P. K. Liaw, J. T. Uhl, R. L. Weaver, and K. A. Dahmen, "Effect of annealing on nanoindentation slips in a bulk metallic glass,” Phys. Rev. B 96, 134117 (2017).

${ }^{24}$ W. C. Oliver and G. M. Pharr, "An improved technique for determining hardness and elastic modulus using load and displacement sensing indentation experiments," J. Mater. Res. 7, 1564 (1992).

${ }^{25}$ W. C. Oliver and G. M. Pharr, "Measurement of hardness and elastic modulus by instrumented indentation: Advances in understanding and refinements to methodology," J. Mater. Res. 19, 3 (2004).

${ }^{26} \mathrm{X}$. Li and B. Bhushan, "A review of nanoindentation continuous stiffness measurement technique and its applications," Mater. Charact. 48, 11 (2002).

${ }^{27}$ J. R. Greer and W. D. Nix, "Size dependence of mechanical properties of gold at the sub-micron scale," Appl. Phys. A 80, 1625 (2005).

${ }^{28}$ A. Clauset, C. R. Shalizi, and M. E. J. Newman, "Power-law distributions in empirical data," SIAM Rev. 51, 661 (2009).

${ }^{29}$ E. K. H. Salje, A. Planes, and E. Vives, "Analysis of crackling noise using the maximum-likelihood method: Power-law mixing and exponential damping," Phys. Rev. E 96, 042122 (2017).

${ }^{30}$ V. Soprunyuk, S. Puchberger, A. Tröster, E. Vives, E. K. H. Salje, and W. Schranz, "Strain intermittency due to avalanches in ferroelastic and porous materials," J. Phys. Condens. Matter 29, 224002 (2017).

${ }^{31}$ V. Soprunyuk, S. Puchberger, W. Schranz, A. Tröster, E. Vives, and E. K. H. Salje, Avalanches in Functional Materials and Geophysics. Understanding Complex Systems: Towards a Quantitative Analysis of Crackling Noise by Strain Drop Measurements (Springer, Cham, 2017).

${ }^{32}$ J. Koppensteiner, W. Schranz, and M. A. Carpenter, "Revealing the pure confinement effect in glass-forming liquids by dynamic mechanical analysis," Phys. Rev. B 81, 024202 (2010).

${ }^{33}$ D. L. Joslin and W. C. Oliver, "A new method for analyzing data from continuous depth-sensing microindentation tests,” J. Mater. Res. 5, 123 (1990).

${ }^{34}$ I. N. Sneddon, "The relation between load and penetration in the axisymmetric boussinesq problem for a punch of arbitrary profile," Int. J. Eng. Sci. 3, 47 (1965).

${ }^{35}$ R. Saha and W. D. Nix, "Effects of the substrate on the determination of thin film mechanical properties by nanoindentation," Acta Mater. 50, 23 (2002).

${ }^{36}$ D. J. O’Brien, T. F. Juliano, P. J. Patel, and S. H. McKnight, "Transparent nanoporous glass-polymer composite for U.S. Army applications," in Carbon Nanotube and Nanocomposite Research, edited by E. Mahler and D. Seiler (Nova Science Pub. Inc., 2011), Chap. 5, pp. 63-78.

37J. L. Gazquez, A. Martinez, and F. Mendez, "Relationship between energy and hardness differences,” J. Phys. Chem. 97, 4059 (1993).

${ }^{38}$ L. Deng, Q. Liu, X. Wang, and J. Li, "Load drop and hardness drop during nanoindentation on single-crystal copper investigated by molecular dynamics," Appl. Phys. A 124, 743 (2018).

${ }^{39} \mathrm{M}$. Hardiman, T. J. Vaughan, and C. T. McCarthy, "A review of key developments and pertinent issues in nanoindentation testing of fibre reinforced plastic microstructures," Compos. Struct. 180, 782 (2017).

${ }^{40}$ W. W. Gerberich, J. C. Nelson, E. T. Lilleodden, P. Anderson, and J. T. Wyrobek, "Indentation induced dislocation nucleation: The initial yield point," Acta Mater. 44, 3585 (1996).

${ }^{41}$ Z. Budrikis, D. F. Castellanos, S. Sandfeld, M. Zaiser, and S. Zapperi, “Universal features of amorphous plasticity,” Nat. Commun. 8, 15928 (2017).

${ }^{42} \mathrm{~J}$. Baró and E. Vives, "Analysis of power-law exponents by maximumlikelihood maps,” Phys. Rev. E 85, 066121 (2012).

${ }^{43}$ G. F. Nataf, P. O. Castillo-Villa, J. Baró, X. Illa, E. Vives, A. Planes, and E. K. H. Salje, "Avalanches in compressed porous $\mathrm{SiO}_{2}$-based materials," Phys. Rev. E 90, 022405 (2014).

${ }^{44}$ Y. Y. Kagan, "Earthquake size distribution: Power-law with exponent $\beta \equiv 1 /$ 2?," Tectonophysics 490, 103 (2010).

${ }^{45}$ A. Ponce-Alvarez, A. Jouary, M. Privat, G. Deco, and G. Sumbre, "Wholebrain neuronal activity displays crackling noise dynamics," Neuron 100, 1446 (2018). 